University of Louisville

ThinkIR: The University of Louisville's Institutional Repository

$5-2013$

\title{
Adherence to the American College of Cardiology/American Heart Association guidelines for pharmacotherapy in New York Heart Association Class II-IV heart failure patients and associated re- hospitalization rates.
}

Matthew T. Mefford

University of Louisville

Follow this and additional works at: https://ir.library.louisville.edu/etd

\section{Recommended Citation}

Mefford, Matthew T., "Adherence to the American College of Cardiology/American Heart Association guidelines for pharmacotherapy in New York Heart Association Class II-IV heart failure patients and associated re-hospitalization rates." (2013). Electronic Theses and Dissertations. Paper 960.

https://doi.org/10.18297/etd/960

This Master's Thesis is brought to you for free and open access by ThinkIR: The University of Louisville's Institutional Repository. It has been accepted for inclusion in Electronic Theses and Dissertations by an authorized administrator of ThinkIR: The University of Louisville's Institutional Repository. This title appears here courtesy of the author, who has retained all other copyrights. For more information, please contact thinkir@louisville.edu. 


\title{
ADHERENCE TO THE AMERICAN COLLEGE OF CARDIOLOGY/AMERICAN HEART ASSOCIATION GUIDELINES FOR PHARMACOTHERAPY IN NEW YORK HEART ASSOCIATION CLASS II-IV HEART FAILURE PATIENTS AND ASSOCIATED RE-HOSPITALIZATION RATES
}

by

\author{
Matthew T. Mefford \\ B.S., University of Louisville, 2007
}

\author{
A Thesis \\ Submitted to the Faculty of the \\ School of Public Health and Information Sciences \\ in Partial Fulfillment of the Requirements \\ for the Degree of \\ Master of Science \\ Department of Epidemiology and Population Health \\ University of Louisville \\ Louisville, Kentucky
}

May 2013 
Copyright 2013 by Matthew Thomas Mefford

All rights reserved 


\section{ADHERENCE TO THE AMERICAN COLLEGE OF CARDIOLOGY/AMERICAN HEART ASSOCIATION GUIDELINES FOR PHARMACOTHERAPY IN NEW YORK HEART ASSOCIATION CLASS II-IV HEART FAILURE PATIENTS AND ASSOCIATED RE-HOSPITALIZATION RATES}

\section{By}

Matthew Thomas Mefford

B.S., University of Louisville 2007

A Thesis Approved on

April 23, 2013

By the following Thesis Committee:

Carlton A. Hornung, Ph.D, M.P.H. -Thesis Director

$$
\text { William McKinnegy/M.D. }
$$

Richard Kerber, Ph.D 


\section{ACKNOWLEDGEMENTS}

I would like to wholeheartedly thank my thesis committee: Dr. Carlton Hornung, Dr. Richard Kerber, and Dr. Paul McKinney, for their patience, insight, and guidance in the preparation of this thesis. I would especially like to thank Dr. Hornung for serving as the director of this committee, and affording me several opportunities to excel academically throughout my graduate career. His mentorship has affirmed and supported my passion for the area of cardiovascular disease epidemiology and clinical research; I wish him well in his retirement and all future endeavors. Additionally, I would like to extend my gratitude to the faculty of the Department of Epidemiology at the University of Louisville for providing a wonderful academic environment in which I could develop a well-rounded epidemiological prowess. 


\begin{abstract}
ADHERENCE TO THE AMERICAN COLLEGE OF CARDIOLOGY/AMERICAN HEART ASSOCIATION GUIDELINES FOR PHARMACOTHERAPY IN NEW YORK HEART ASSOCIATION CLASS II-IV HEART FAILURE PATIENTS AND ASSOCIATED RE-HOSPITALIZATION RATES
\end{abstract}

Matthew T. Mefford

April 24, 2013

This thesis will assess differences in the rates of rehospitalization among New York Heart Association class II, III, and IV heart failure patients, focusing on levels of adherence to the American College of Cardiology/American Heart Association guidelines for heart failure patient pharmacotherapy. Hospitalization data on $128 \mathrm{HF}$ patients will be analyzed looking at 14 day, 30 day, and 6 month time points, assessing key aspects of treatment and patient characteristics as potential risk factors in predicting rehospitalization trends. Effectiveness of ACC/AHA adherence to pharmacotherapy upon primary hospital discharge will be itemized in an intention to treat analysis, with guidelines determined by compliance between NYHA classification and ACC/AHA recommendations. Confounding interactions by demographic characteristics including race, sex, and age will be included. This study will gauge the effectiveness of current HF patient care criteria as well as point out potential confounding effects associated with prescribed care previously unaccounted for in the current literature. 
TABLE OF CONTENTS

PAGE

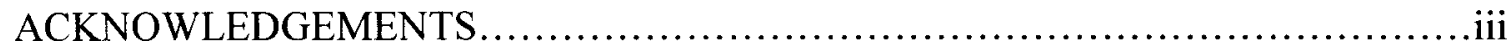

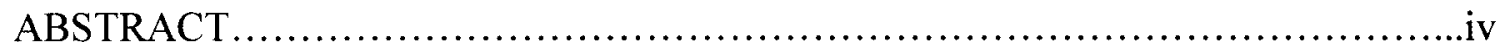

INTRODUCTION

Epidemiology of Heart Failure................................................

Pathophysiology/Etiology of Heart Failure..................................6

Classification and Staging of Heart Failure......................................10

Pharmacotherapy of Heart Failure .........................................15

RESEARCH

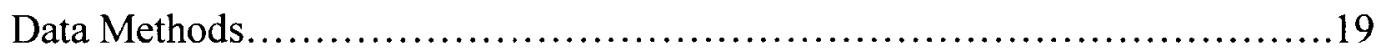

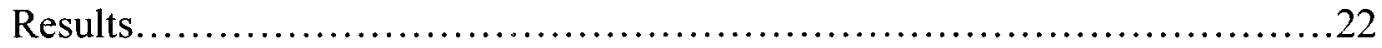

Hazard Plots........................................................ 27-30

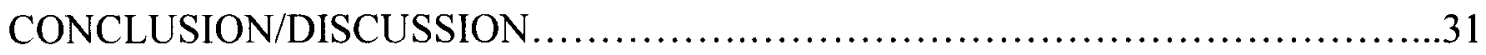

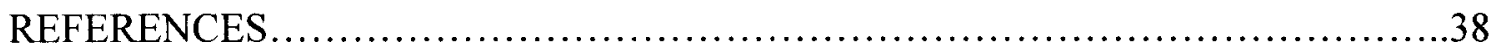

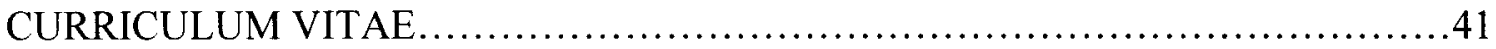




\section{EPIDEMIOLOGY OF HEART FAILURE:}

Heart failure rates, mortality, prevalence, and incidence have been classified by several different research studies, each with variances in the populations studied and data collection methods. For example, the Framingham study - which began in 1948 - had a list of symptoms, physical findings, and physiological measurements used to classify congestive heart failure; classification as a case required multiple criteria be met, with no other medical explanation than heart failure for the presence of "minor" criteria. ${ }^{17}$ In contrast, the development of ACCF/AHA guidelines re-defined HF as a clinical syndrome characterized by symptoms in the medical history of an individual as well as signs in a physical examination. With no single diagnostic test for presence of HF, most diagnoses are subjective and based on qualitative judgment. Interpretation of epidemiological data of HF is compromised by these variances. Because there is not a universal classification for heart failure, this limitation should be borne in mind when considering the following data presented.

The National Health and Nutrition Examination Survey (NHANES) is a program of studies designed to assess the health and nutritional status of adults and children in the US and is a combination of both physical examinations of interviews. ${ }^{6}$ Results of the NHANES 2005-2008 estimated that 5.7 million Americans over the age of 20 years have

HF. ${ }^{26}$ Projections on crude prevalence from 2010 show that approximately 6.6 million Americans ( $2.8 \%$ of the population) had HF, with the trend increasing each year. It's 
estimated that by 2030 , with trends continuing upward, an additional 3 million people will have $\mathrm{HF}$, a $25 \%$ increase in prevalence from $2010 .{ }^{27}$ Annual rates per 1000 population of new HF events for white men are as much as 15.2 for those 65 to 74 years, 31.7 for those 75 to 84 , and 65.2 for those greater than 85 years; for white women in the same age groups, rates are 8.2, 19.8, and 45.6. For black men, rates are 16.9, 25.5, and 50.6, and for black women, rates are 14.2, 25.5, and 44.0. (CHS, NHLBI)

The Multi-Ethnic Study of Atherosclerosis (MESA) was a medical research study on cardiovascular disease risk factors that involved more than 6,000 men and women from six communities in the US and was funded by the NHLBI and NIH. Data from MESA ranked the risk of developing HF by all ethnic groups in the following order: African American, Hispanic, white, and Chinese American (4.6, 3.5, 2.4, and 1.0 per 1000 person-years, respectively). ${ }^{27}$ These differences in risk can be directly correlated with prevalence of hypertension and other comorbid conditions, as well as socioeconomic status (SES). African Americans also had the highest proportion of incident HF not proceeded by clinical myocardial infarction (MI) ${ }^{27}$ In the ARIC study, age adjusted incidence of HF per 1000 person-years was 9.1 for black men (BM), 8.1 for black females (BF), 6.0 for white men (WM), and 3.4 for white females (WF). The 30-day, 1 year, and 5 year case fatality rates after rehospitalization for $\mathrm{HF}$ were $42.3 \%$ in BM, $22 \%$ in BF, and $10.4 \%$ in WM. ${ }^{19}$ Similarly, the CARDIA study, looking at CVD differences in young black and white adults, concluded that HF before the age of 50 years was more common among black individuals. Hypertension, obesity, and systolic dysfunction were all considered important risk factors in the development of disease and targets for prevention. ${ }^{27}$ 
Risk factor epidemiology for HF patients shows a distinct group of lifestyle conditions that critically affect the severity of disease onset and progression. The Framingham study listed lifestyle-related factors increasing risk of HF including cholesterol concentration, smoking status, blood pressure, and diabetes; presence of left ventricular hypertrophy $(\mathrm{LVH})$ as a consequence of hypertension - resulting in an enlargement of the left ventricle - as determined by electrocardiography (ECG) is also considered a significant risk factor. ${ }^{17}$ Older males and females had a higher relative risk for the development of HF with the presence of hypertension, diabetes, and LVH: 1.9, 2.0, and 4.9, respectively, in males, and 1.9, 3.6, and 5.4 in females. Men aged 35-64 also had a significantly increased risk of HF development if they were smokers $(\mathrm{RR}=1.5) .{ }^{17}$ Among 20,900 male physicians in the Physicians Health Study, the lifetime risk of HF was higher in men with hypertension; healthy lifestyle factors (normal weight, not smoking, regular exercise, moderate alcohol intake, etc) were related to lower risk of HF. ${ }^{27}$ Among the participants of the Health Aging, Body and Composition (ABC) study, there was a higher proportion of HF attributable to modifiable risk factors in black participants over white participants $(67.8 \%$ vs. $48.9 \%)$; in this study, inflammatory markers were also attributed to HF risk. ${ }^{27}$

In 2008 , overall death rate for $\mathrm{HF}$ was 84.6 per 100,000 individuals; the rates listed by varying race/sex class were listed as 98.9 for WM, 102.7 for BM, 75.9 for WF, and 78.8 BF. (NCHS, NHLBI) Any-mention mortality with HF was 281, $437(124,598$ males and 156,839 females) with HF as the major underlying cause in 56,830 of those deaths in 2008. Additionally, 1 in 9 deaths had HF listed on the death certificate. ${ }^{27}$ Survival after HF diagnosis has improved over time, as shown by data in the Framingham 
Heart Study (FHS) and the Olmsted County study. However, death rate remains high approximately $50 \%$ of people diagnosed with HF will die within an average of 5 years. ${ }^{27}$ A unique aspect of HF mortality is the proportion of deaths that are sudden. The FHS predicted that between $40-50 \%$ of deaths in the presence of HF were sudden, defined as occurring within one hour in a previously stable patient. ${ }^{17}$ When looking at 30 year follow up in the Framingham population, the presence of previous HF increased the risk of sudden death as much as sevenfold when CHD was absent and nine-fold in the presence of CHD. ${ }^{17}$

Data from Kaiser Permanente suggest that survival after the onset of HF has improved in elderly populations, although mortality rates are still high. ${ }^{2}$ For women ages $65-74,15$ year mortality rates were approximately $60 \%$; the corresponding rate of men in the same age group was even higher. ${ }^{17}$ This improvement seen over time can be attributed to advancements in surgical and treatment approaches in HF management ${ }^{17}$. The one area in which rates do not improve for heart failure patients lies in the hospitalization and rehospitalization data. From 1999 to 2009, hospital discharges for HF were virtually unchanged, with first-listed discharges of 975,000 and 1,094,000 respectively. (NHDS 2009, NCHS, NHLBI)Despite the insignificant change in discharge rates, rate of hospitalization for HF has increased more than threefold over the past three decades, with the increasing pattern largely being attributed to elderly populations. One age group where a positive trend is also witnessed is in the 45-64 year age group. ${ }^{17}$ With this said, the overall case-fatality rate has substantially declined by more than $50 \%$ through the 1980's and 1990's. ${ }^{17}$ 
The amount of heart failure treatment paints an even larger picture of the overall burden of the disease, with HF imposing a large economic burden on the health care system. In developed countries, the cost of HF care constitutes approximately $1 \%$ to $2 \%$ of overall health care spending. ${ }^{9}$ In 2009 , the estimated direct and indirect cost of treating HF patients in the United States was more than $\$ 37$ billion, this being the largest consumer of Medicare spending above any other diagnosis. ${ }^{4}$ Alarmingly, this figure may increase in following years due to the aging population and increased survival after primary hospitalization. Elderly patients, often covered by Medicare, are rehospitalized more frequently than younger HF patients $-25 \%$ being rehospitalized in the first 30 days; Medicare, however, does not reimburse hospitals for this time period shifting the cost burden to the healthcare facility.

In a study looking at the lifetime medical costs of HF patients in Olmsted County, Minnesota, 1054 patients were randomly sampled and assessed for total accrued debts ${ }^{9}$. Total costs for the cohort during the study period (1987 to 2006) were estimated at $\$ 100$, 967,086 in 2006, combining both inpatient and outpatient costs. ${ }^{9}$ The majority of costs were due to hospitalizations ( $77 \%$ ) with an average cost of $\$ 73,762$ per person. The highest proportion of inpatient costs were rooming, procedures, and evaluation and management. Total outpatient costs accounted for over $\$ 22$ million, with an average cost of $\$ 22,032$ per person. ${ }^{9}$ The highest proportion of outpatient cost was due to evaluation and management and procedures. Among evaluation and management, office visits comprised the largest amount of cost. Dialysis, if needed, was the largest proportion of procedural cost. Lifetime costs were estimated for all patients at approximately $\$ 109,541$ 
per person, with comorbid conditions such as diabetes increasing estimated costs over $30 \%{ }^{9}$

Although overall trends are decreasing for incidence of HF, the cost burden associated with the management of heart disease is highly significant. In 2009 , the US spent $\$ 2.6$ trillion on health care, representing more than $17.6 \%$ of our gross domestic product. ${ }^{9}$ Although annual costs of caring for the populations affected by HF in the US are estimated as being high, little is known about the cumulative costs of caring for HF patients after diagnosis. Some estimates suggest that over $50 \%$ of the total costs were accumulated within the last 6 months of the individual's life. ${ }^{9}$ Identifying patient factors associated with higher costs may be helpful in targeting those patients for cost-saving interventions. Additionally, standardizing treatment practices among physicians and medical institutions may improve patient outcomes and reduce the total cost associated with this disease.

\section{PATHOPHYSIOLOGY/ETIOLOGY OF HEART FAILURE:}

Heart failure (HF) is a chronic, progressive condition in which the heart muscle is unable to pump enough blood to meet the body's needs for oxygen and nutrients. ${ }^{1}$ In order to compensate for this reduction in blood flow and increase in demand from surrounding body tissue, the heart will typically undergo one of the following: enlargement, increase in muscle mass, or increased pumping action. The body in addition will compensate in other ways: blood vessels can narrow to maintain blood pressure (making up for the reduction in pumping action/power), or divert blood from less important tissues and organs to maintain flow to the vital organs (i.e. the heart and 
brain). ${ }^{1}$ While these temporary solutions can delay the outcome of total heart failure, conditions will worsen until these substitute process no longer work, or some type of medical intervention occurs. Once an individual's body compensation cannot keep up with the energy demands it he/she will experience fatigue, breathing problems, or any myriad of life threatening symptoms - the most severe being death.

HF primarily reflects the impairment of the pumping function of the heart's left ventricle, although other etiologies exist. ${ }^{17}$ The result of this impairment is a reduction in blood flow from the left ventricle to the aorta, which in turn reduces peripheral arterial blood flow. In LV failure, the left side of the heart must exert a larger amount of energy to pump a normal amount of blood. This increase in exertion often leads to a hardening of the left ventricular wall ("stiffness") or enlargement of the ventricle (left-ventricular hypertrophy). Additionally, failure to eject blood from the left ventricle leads to an increased back pressure in the pulmonary circulation, accompanying and exacerbating respiratory dysfunction. Heart failure can occur on either the left or right side, with each classification having distinguishing impairments. Mechanisms of left heart failure include "systolic" and "diastolic" dysfunction. Systolic dysfunction describes a heart with diminished ejection fraction, where the left ventricle expels a reduced amount of blood at each contraction of the myocardium; diastolic dysfunction describes an incomplete relaxation of the left ventricle (typically due to stiffness of the ventricular wall), resulting in a reduced volume of blood entering the chamber during each relaxation. ${ }^{17}$

The heart moves deoxygenated blood that returns to the heart through the veins through the right atrium into the right ventricle. The ventricle then pumps blood back out of the heart into the lungs to be replenished with oxygen. Right-sided heart failure occurs 
due to an impairment of right-ventricular (RV) function, and occurs as a result of leftsided, LV failure.' Increased fluid pressure in the lungs, which often times occurs due to left-sided failure, damages the right ventricular tissue. When the right side loses power, blood backs up in the body's veins, typically resulting in peripheral edema in the legs and ankles; right failure also occurs in conjunction with obesity or sleep-disordered breathing. ${ }^{17}$

A myriad of conditions can damage or weaken the heart and cause heart failure. The most common form of heart disease and the a leading cause of HF is coronary artery disease (CAD). ${ }^{20} \mathrm{CAD}$ is one of several terms referring to atherosclerosis, or narrowing of the arteries that supply blood to the heart due to a build-up of fatty deposits. This slowing of blood flow through the body leaves areas of the myocardium weak and oxygen deprived. In most cases, however, the reduction in blood flow is not enough to fully impair the heart muscle, but sufficiently weaken cardiac output; if plaque build-up occludes the arteries to the point in which myocardial cells are deprived of blood flow (ischemia), weakening or death of cell tissue occurs. When these plaques rupture, blood clots block blood flow to an area of the heart muscle, weakening the heart's pumping ability. ${ }^{20}$

Other contributing factors to heart failure include hypertension, heart valve dysfunction, cardiomyopathy, myocarditis, congenital heart defects, heart arrhythmias, and other diseases. Hypertension, one of the most common chronic conditions related to vascular health (affecting more than 70 million Americans), is a critical measure of circulatory function. ${ }^{17}$ It reflects a balance between the blood volume ejected from the left ventricle of the heart during each contraction and the arterial resistance to blood flow. 
Several physiologic mechanisms operate to simultaneously maintain blood pressure based on the perfusion needs of surrounding body tissues. Hypertension, however, causes the heart to work harder than normal to pump blood throughout the body, which leads to thickening of the myocardium to compensate for the extra work it must perform. Over time, the cardiac muscle becomes either too stiff or too weak to effectively pump blood. ${ }^{20}$ Valve damage, typically due to a heart defect, $\mathrm{CAD}$, or infections, forces the heart to overexert to keep blood flowing in the normal direction. Myocarditis, an inflammation of the heart muscle (commonly caused by infection) can also lead to left-sided failure.

Congenital heart defects, those present at birth, are a product of improper development/formation of the chambers or valves of the heart. As a result, the healthy, developed portions of the heart have to increase work load to pump blood through the heart, which can in turn lead to heart failure. In addition, abnormal heart rhythms, or arrhythmias, can cause the heart to beat too quickly. This too creates extra work for the heart to pump blood correctly. A slow heart beat may prevent the myocardium from pushing enough blood out to the body; both scenarios cause a weakening of the myocardium and can lead to HF. Chronic conditions such as diabetes, anemia, hyperthyroidism, hypothyroidism, emphysema, lupus, or a build-up of iron, protein, or inflammatory cells can also contribute to heart failure. ${ }^{20}$ Finally, acute heart failure can be brought on by viruses that attack the myocardium, severe infection, allergic reactions, blood clots in the lungs, and certain medications. ${ }^{20}$ 


\section{CLASSIFICATION AND STAGING OF HEART FAILURE:}

The New York Heart Association (NYHA) classification system is one of the oldest attempts to grade the severity of functional limitation among heart failure patients. Initially developed in 1928, this classification scheme, although modified since its inception, has withstood the test of time and is still widely used by physicians today; its accuracy, however, is widely debated. The scale is divided into four functional groups (class I - least severe to class IV - most severe), separated by a series of qualifying descriptions related to an individual's physical ability. To accurately assess the extent of functional limitation, the NYHA committee prescribes using a series of exercise tests to determine the extent to which patients with impaired or limited cardiac function can perform without discomfort. The determination of physical impairment spans from class I patients, who exhibit no limitation in physical activity, to class IV patients, who experience discomfort performing any physical activity. Full descriptions of the four classes are listed as such:

New York Heart Association (NYHA) Functional Classification for Heart Failure Patients:

\begin{tabular}{|l|l|}
\hline NYHA Class & Symptoms \\
\hline I (Mild) & $\begin{array}{l}\text { No limitation of physical activity. Ordinary physical } \\
\text { activity does not cause undue fatigue, palpitation, or } \\
\text { dyspnea. }\end{array}$ \\
\hline II (Mild) & $\begin{array}{l}\text { Slight limitation of physical activity. Comfortable at } \\
\text { rest, but ordinary physical activity results in fatigue, } \\
\text { palpitation, or dyspnea. }\end{array}$ \\
\hline III(Moderate) & $\begin{array}{l}\text { Marked limitation of physical activity. Comfortable at } \\
\text { rest, but less than ordinary activity causes fatigue, } \\
\text { palpitation, or dyspnea }\end{array}$ \\
\hline IV (Severe) & $\begin{array}{l}\text { Unable to carry out any physical activity without } \\
\text { discomfort. Symptoms of cardiac insufficiency at rest. } \\
\text { If any physical activity is undertaken, discomfort is } \\
\text { increased. }\end{array}$ \\
\hline
\end{tabular}


Although widely respected, the guidelines to determine these classifications are subjective, with no set physical examination guidelines or standards; there are an endless number of physical exertion tests one can perform to come to the same conclusion. Additionally, there are no set guidelines to distinguish different levels of ability, with terms like "slight" or "marked" leaving the interpretation of limited physical ability up to the physician. Common criteria used to determine NYHA class include: self-reported walking distance, difficulty climbing stairs, ability to walk to local landmarks, breathlessness interfering with daily activity, and breathlessness when walking around the house. ${ }^{26}$ While some methods (such as walking distance) are more widely used than others not one distinct assessment has been accepted as superior to others, although the ACC has distinct parameters for classification ; single clinics creating original classification schemes often protect their intellectual property from being used across multiple institutions. This inconsistency is apparent in the published literature, with $99 \%$ of research papers withholding a reference or description of their methods for assigning NYHA class in subjects. ${ }^{26}$

However subjective the classification system, the NYHA scheme is simple enough to provide a measure of the functional capability in heart failure patients and allow physicians to relay immediate feedback to these individuals. However one decides its magnitude of importance, it has no basis in the prescription of medication or course of treatment. Rather it allows a physician to gauge the extent of disease progression without medical testing. For more extensive criteria on the classification of heart disease, other organizations have created guidelines that are widely used today. The best example heart 
failure treatment guidelines are those formulated by the American College of Cardiology and the American Heart Association, who in a joint task force issued the Guidelines for the Evaluation and Management of Chronic Heart Failure. These guidelines offer a comprehensive determination scheme involving both determination of disease progression and level of evidence in suggesting pharmacotherapies.

The ACC/AHA first published guidelines for the evaluation and management of HF patients in 1995, and a great deal of progress has been made since its inception both in the area of pharmacological and non-pharmacological approaches to treatment. Although the most current update of guidelines occurred in the year 2009, for purposes of this thesis the guidelines presented in 2001 will be used; this will maintain continuity with the time frame of the study data, which was collected in 2004-2005. The committee composed of 7 members representing the ACC and AHA, identified 4 stages of HF, similar yet distinguishable from the NYHA classification. Stage A classifies patients who have a high risk for developing HF, but show no structural disorder of the heart; Stage B refers to a patient with a structural disorder of the heart but who not yet developed symptoms of HF; Stage C classifies a patient with past or current symptoms of HF associated with underlying structural heart disease; Stage D denotes patients with endstage disease who require specialized treatment strategies. ${ }^{14}$ Only the latter two stages, according to clinical diagnostic guidelines, qualify as HF. In comparing to the NYHA guidelines, class I-IV would only classify those individuals in stage C or D of the AHA/ACC guidelines. ${ }^{14}$

In contrast to the NYHA classification system, the ACC/AHA developed classes (in addition to stages of disease) that correspond to levels of evidence on treatment 
protocol, and provide recommendation on the appropriate course of treatment to prescribe given the progression of heart disease. Class I describes a condition for which there is evidence and/or general agreement that the procedure or treatment is useful and effective; Class II describes a condition for which there is conflicting evidence and/or a divergence of opinion about the usefulness/efficacy of a procedure or treatment; Class Ila describes a condition in which the evidence or opinion is in favor of the procedure or treatment; Class IIb describes a condition for which usefulness or efficacy less well established by evidence or opinion; and Class III describes conditions for which there is evidence and/or agreement that the procedure or treatment is not useful/effective and in some cases can be harmful. ${ }^{14}$ Each class has levels of evidence indicated by a grading scale of A, B, and C. Levels of evidence are ranked as $\mathrm{A}$ if the data is derived from multiple randomized clinical trials, B when data were derived from a single randomized trial or nonrandomized studies, and $\mathrm{C}$ when the consensus opinion of experts was the primary source of recommendation. ${ }^{14}$ The table showing the various classes and levels of evidence is listed below: 


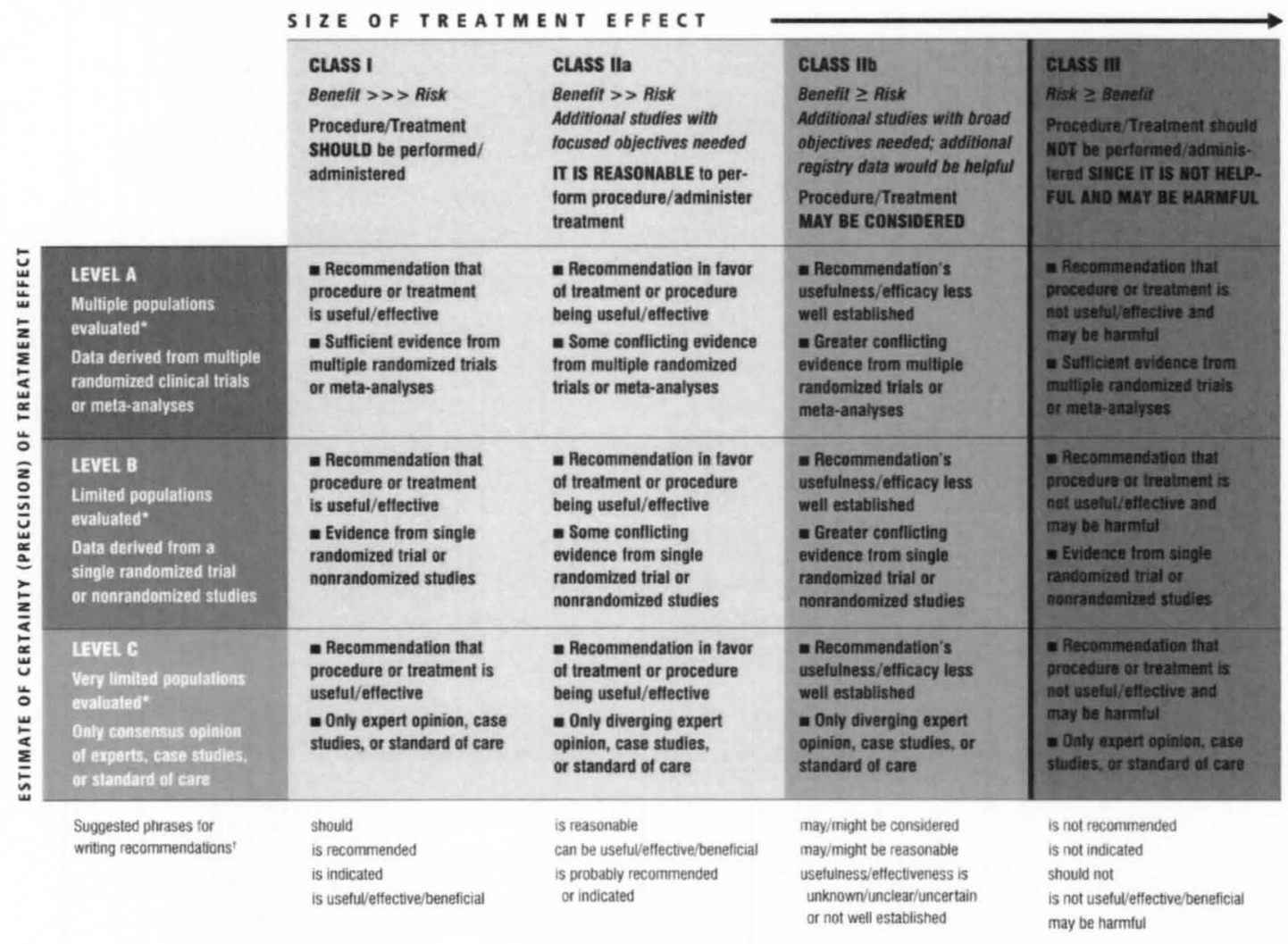

Source: American Heart Association/American College of Cardiology Treatment Guidelines Update, 2001

The ACC/AHA guidelines clearly lay out treatment recommendations based by stage, class, and level of evidence. Its advantage over the classification system of the NYHA lies in that it objectively identifies patients in the course of their disease and links them to treatment appropriate at each stage of their disease; the NYHA system, in contrast, offers little variation in treatment recommendation as it subjectively classes subjects based on non-clinical assessment. ${ }^{14}$ In addition, the ACC/AHA approach expects that each patient advances from one stage to the next until progression is slowed or stopped by treatment. ${ }^{14}$ Despite the inconsistencies between the two systems, they both remain invaluable in the classification and treatment of HF patients, as modeled by research on the relationship between class and outcome (with NYHA guidelines) and treatment, class, and outcome (with ACC/AHA guidelines). 


\section{PHARMACOTHERAPY OF HEART FAILURE:}

\section{ANGIOTENSIN CONVERTING ENZYME (ACE) INHIBITORS:}

Of the myriads of medications prescribed to patients hospitalized with heart failure, angiotensin-converting enzyme (ACE) inhibitors are arguably the most widely used in the course of treatment. ACE inhibitors function by relaxing the blood vessels and are primarily used in the treatment of hypertension. Biologically, the mechanism involves ACE inhibitors preventing enzymes in the body from producing angiotensin II a hormone that affects the cardiovascular system by narrowing blood vessels and releasing hormones that raise blood pressure - forcing an increase in cardiac output. ${ }^{20}$ Commonly prescribed examples of ACE inhibitors include: benazepril, captopril, and enalapril. To determine the importance of ACE inhibitors outside of hypertension therapy, several randomized clinical studies have assessed the role of ACE inhibitors in patients with acute myocardial infarction (AMI) or HF.

An analysis of three trials using HF patients enrolled within a week of HF hospitalization and randomly assigned to either treatment or placebo groups modeled the effect of ACE inhibitors. Of the 12,763 patients in these studies, individuals prescribed ACE inhibitors had overall lower mortality $(\mathrm{OR}=0.74 ; 95 \% \mathrm{CI} 0.66-0.83)$ relative to the placebo group. Rehospitalization rate, reinfarction rate, and composite of all these events were also lower. ${ }^{27}$ Additionally, these benefits were independent of age, sex, and other factors that commonly contribute as competing factors in the effects model. Other studies have focused on specific populations of individuals to model the effects of ACE inhibitors. Looking at the effects of these medications in older individuals, an average 
reduction in mortality (approximately 10\%) was found among ACE inhibitors users relative to use of other medications such as Digoxin. ${ }^{13}$

\section{ANGIOTENSIN RECEPTOR BLOCKER (ARB):}

Angiotensin Receptors Blockers (ARBs), also known as angiotensin II receptor antagonists are commonly prescribed medications in the treatment of hypertension. Similar in effect to ACE inhibitors, but alternatively prescribed due to ACE-I intolerance, they have also proven effective in the treatment of heart failure. At the most basic description, ARBs function by relaxing the blood vessels and lowering blood pressure, allowing the heart to pump blood with greater ease. From a biological perspective, the mechanism of angiotensin receptor blockage directly affects the renin-angiotensinaldosterone system (RAAS) by reducing the secretion of vasopressin and reducing the production and secretion of aldosterone. Commonly prescribed examples of ARBs include: candesartan, eprosartan, and losartan. Studies evaluating the role of ARBs in HF treatment, although less abundant than those focusing on ACE inhibitors, provide insight on their effectiveness relative to other therapies.

In a randomized trial evaluating HF patients receiving ARBs or placebo throughout the course of study, combined end point mortality was lower among those receiving $\mathrm{ARB}(\mathrm{RR}=0.87 ; \mathrm{CI}(0.77-0.97)$. Treatment with $\mathrm{ARB}$ also improved ejection fraction, NYHA class, and reported quality of life. ${ }^{7}$ The ELITE study, evaluating the effectiveness of ARBs in an elderly population of HF patients, showed a risk reduction of $32 \%$ among those using the medication relative to ACE-inhibitor use, primarily due to a decrease in all-cause mortality; hospitalization rates were less frequent among ARB 
users, as well. ${ }^{24}$ ARB prescription is a common alternative to ACE-inhibitors due to their lack of adverse effects in patients. The ELITE-II study, in follow-up to the original study, aimed to determine if one course of treatment was superior to the other (ACE-I vs. ARB). In the population studied (not just the elderly, as in ELITE) while mortality and sudden death rates remained similar, the discontinuation of treatment in the ARB group due to adverse effects was significantly lower than in the ACE-I group $(9.7$ vs. $14.7, p<0.001) .^{23}$ Overall, the study on efficacy of these two treatments has proven that either medication is beneficial in the pharmacotherapy of HF patients.

\section{BETA-BLOCKERS:}

Beta adrenergic receptor antagonists, commonly known as beta-blockers, are a class of drugs that block the effects of epinephrine on the beta receptors found in smooth muscle, airways, arteries, kidneys, and most importantly, the heart; beta-blockers reduce blood pressure by negating the effect of epinephrine, thereby reducing heart rate. Betablockers have proven effective in reducing overall mortality among HF patients, as well as improve LV function. ${ }^{8}$ In a meta analysis of randomized trials involving patients with congestive heart failure, there was a greater than $30 \%$ reduction in odds of death among patients assigned a beta-blocker (CI 11-46\%), representing an absolute reduction in mean annual mortality from $9.7-7.5 \% .^{8}$ Carvedilol, the most commonly prescribed betablocker, has been proven to effectively reduce mortality among patients with moderate to severe HF. In a random trial of HF patients (NYHA class III or IV) mortality was reduced by $35 \%$ among patients prescribed carvedilol, with an almost $25 \%$ reduction in combined mortality. ${ }^{22}$ Beta-blockers usage among moderate HF patients showed an average death rate of $8 \%$ compared to $12.8 \%$ among non-users $(\mathrm{OR}=0.63,95 \% \mathrm{CI} 0.55$ - 
0.72); similar reductions were observed for hospital admissions for worsening HF, and composite outcome of death. ${ }^{28}$

\section{SPIRONOLACTONE:}

Spironolactone is commonly used in combination with other medications (such as those listed previously) to treat hypertension and HF. Although a relatively old medication, introduced clinically in 1959 , its importance in HF management and treatment is often understated, as exhibited by a low level of evidence in ACC/AHA guidelines for severe HF. In the RALE study of severe HF patients, a relative reduction of $30 \%$ was witnessed among those prescribed spironolactone as opposed to placebo $(\mathrm{RR}=0.70 ; 95 \% \mathrm{CI} 0.60-0.82)$. This significant reduction was attributed to lower risk of death from progressive heart failure and sudden death from cardiac causes. Additionally, hospitalization rates were reduced by $35 \%$ and patients had a significant improvement in the symptoms of heart failure as assessed on the basis of their NYHA functional class. ${ }^{25}$ Spironolactone added to ACE-inhibitors has been proven beneficial to improve heart dysfunction, as well. ${ }^{3}$ Where the RALE study proved treatment was beneficial in patients with severe HF, data to support the beneficial effects in all HF patients remains inconclusive. Some research suggests an increase in adverse outcomes associated with spironolactone use when the RALE study guidelines for patient evaluation are not met. ${ }^{5}$ 


\section{DATA AND METHODS:}

The data used in this thesis was derived from "Home HF care comparing patientdriven technology models", an AHRQ funded and IRB approved clinical study. This parallel, randomized, multi-site prospective cohort study assessed the impact of health information technologies on clinical and financial outcomes for patients with symptomatic (NYHA class II - IV) heart failure (HF). The information technologies include remote monitoring (telemonitoring) of vital signs and symptoms, an electronic health record system, and clinical decision support systems. Two different configurations of health information technology were used: technology supported case management, a combination of telemonitoring and telephone nurse case management, and computerbased self management, which combined telemonitoring with an expert clinical decision support system that assessed vital signs and symptoms for risk of decompensation. (Goldberg, 2004) Patients were recruited from both urban and rural areas, including southern Montana, northern Wyoming, Philadelphia, and southern New Jersey; these geographic regions included Native American, African American, Caucasian, and Hispanic HF patients.

In total, 134 patients were recruited for the initial study. The index event for each patient was the date of primary hospitalization for HF. Patients were enrolled within 18 months of their primary hospitalization date and evaluated over the span of one year after enrollment for subsequent hospitalization dates. Reasons for subsequent hospitalization 
included heart failure, cardiovascular-related causes, and non-CV-related causes (classified as "all cause"). In this analysis, if patients upon their first rehospitalization were classified as "all cause" but had a later rehospitalization for CV or HF-related causes, they were classified in the $\mathrm{CV} / \mathrm{HF}$-rehospitalization group with the first rehospitalization date being used to calculate their time-to-event; this assumes that the first rehospitalization could be attributed to an underlying cardiovascular deficiency which would lead to subsequent heart failure. Of additional importance to this analysis were specific periods of time in which subsequent hospitalization occurred, including: 014 days, 15-30 days, 31-180 days, and greater than 180 days. The rate of rehospitalization is important to medical institutions treating HF patients, with respect to the bylaws of the Affordable Care Act (this will be discussed in a later section). Six patients in total were excluded from the analysis: 2 were excluded for having less than 1 month of follow-up, and 4 were excluded for having no index event (i.e., were admitted directly to an emergency room) - this made the final patient count 128 .

Demographic variables including age, sex, and race were recorded for all patients; data on education, marital status, and living situation were incomplete in some patients as this information was self-reported. In addition, comorbid conditions and treatment therapies were recorded for each patient. Comorbid conditions - assessed at enrollment included obesity (BMI>30), hypertension, hyperlipidemia, hypercholesterolemia, diabetes I and II. Obesity was coded binomially, while the remaining comorbid conditions were coded as "doesn't have" - 1, "does have" - 2, or "not determined" - 3; those with a non-determined status were based on incomplete hospital data. Smoking status was classified in 4 categories: "never smoked" - 1, "used to smoke" - 2 , "currently 
smokes" -3 , and "not determined" -4 . Treatment therapies included prescription of ACE inhibitors, ARBs, Beta-blockers, Spironolactone, as well as the total number of prescriptions each patient had been prescribed at enrollment; all treatment variables were categorized binomially as either "prescribed" or "not prescribed" $(1$ or 0$)$ with the exception of number of total prescriptions, which was a continuous variable.

The compliance variable gauging ACC/AHA treatment guidelines for each NYHA class was based on an all-or-none approach ( 0 for non-compliance and 1 for compliance), based on the recommendations for clinical practice put forth from the AHA; partial compliance was not used due to limited information in the original study set with regard to baseline clinical measures, which would have accurately provided conditional situations for modified treatment. In cross-relating the two classification systems, Class II and Class III in the NYHA corresponded with stage C heart failure patients of the AHA guidelines. Class IV in NYHA classification corresponded with stage D heart failure patients of the AHA guidelines. Compliance criteria are listed below, and are based on the 2001 ACC/AHA guidelines for treatment therapy:

NYHA Class II: ACE inhibitor or ARB

NYHA Class III: ACE-I or ARB and Beta-blocker (Spironolactone prescribed if LVEF $<40 \%$ )

NYHA Class IV: ACE-I or ARB, Beta-blocker, and Spironolactone

The guidelines used in this analysis, although outdated by additional updates from the ACC/AHA, correspond with the time frame in which the study was conducted; from this, 
the assessment of compliance was accurate in the study data. The most recent guidelines issued in 2012 remain similar in treatment recommendations based on levels of evidence.

\section{RESULTS:}

The goal of this research analysis was to compare trends in re-hospitalization and event rates among patients with a primary hospitalization for $\mathrm{HF}$, looking at various factors including age, race, sex, NYHA class, and living situation, as well as comorbidities (ex: hypertension, hypercholesterolemia, hyperlipidemia, diabetes, obesity). Outside of analyzing treatment guideline adherence, it is important to look at multiple factors that can affect an individual's health status, especially studying heartrelated illness. In order to obtain an accurate picture of the health of the individuals in this study population, it is important to assess their health characteristics at various stages of heart failure. Descriptive statistics of the study population stratified by their NYHA functional classification is listed in the table 1.

Table 1. Descriptive Statistics for each NYHA class

\begin{tabular}{|l|l|l|l|}
\hline NYHA Class & II & III & IV \\
\hline Mean Age & 66 & 66 & 64 \\
\hline Sex (male) \% & 54 & 29 & 36 \\
\hline Race \% & $84(\mathrm{C}), 15(\mathrm{AA})$ & $69(\mathrm{C}), 22(\mathrm{AA})$ & $79(\mathrm{C}), 14(\mathrm{AA})$ \\
\hline BMI $>30 \%$ & 49 & 58 & 64 \\
\hline Smoker (ever) \% & 27 & 44 & 52 \\
\hline Hypertension \% & 75 & 80 & 93 \\
\hline Hypercholest. \% & 36 & 17 & 14 \\
\hline Hyperlipidemia \% & 44 & 41 & 57 \\
\hline DM \% & 36 & 34 & 79 \\
\hline Lives alone \% & 33 & 39 & 36 \\
\hline CV/HF rehosp. \% & 22 & 44 & 43 \\
\hline $\begin{array}{l}\text { ACC/AHA } \\
\text { compliance \% }\end{array}$ & 76 & 56 & 29 \\
\hline
\end{tabular}


With the exception of mean age, each NYHA class group of patients has distinct differences. Of note, proportion of females, average BMI, smoker status, hypertension, hyperlipidemia, and diabetes mellitus all increase with increase in NYHA class. The comorbid conditions listed provide evidence of the deteriorating overall health of the patient, which may contribute to a decline in cardiovascular health; conversely, the deteriorating condition of the heart may provide a direct influence on any of these several conditions, most notably hypertension. One notable decline with an increase in NYHA class is ACC/AHA compliance, with $76 \%$ compliance in the NYHA class II group, $56 \%$ in the class III group, and only $29 \%$ in the class IV group (which should be receiving the most careful treatment of all patients).

Overall rehospitalization rate within the study period was $59 \%$, translating to 76 total patients; of these, 44 patients were re-hospitalized with a primary or secondary diagnosis for HF (secondary diagnosis was defined as any cardiovascular-related hospitalization). In the evaluation of guideline compliance for treatment therapies, $44.9 \%$ (22/49) of patients were rehospitalized for HF when ACC/AHA guidelines were NOT met, compared to $27.8 \%(22 / 79)$ among those patients in which guidelines for treatment were fulfilled. A risk reduction was exhibited among all individuals over the study period ( 1 year) when treatment guideline adherence was met. This decreased risk across the entire study period provides a baseline trend for the benefit of compliance, although variations exist at different time periods (listed in table 2).

To estimate the effect of time on risk of rehospitalization more precisely, relative risks were calculated for following time intervals: 0-14days, 15 days-1 month (30 days), 31 days to 6 months (180days), and cumulative 30 day; cumulative risk was also 
calculated for the entire study period. Relative risks for age $(\geq 65$ years vs. younger than 65 years), race (white vs. non-white), and sex (male vs. female) were calculated for each time interval, as well as the relative risk for NYHA class (class IV vs. II/III) and ACC/AHA compliance variables. Each relative risk calculated was controlled for study arm to account for treatment differences patients may have received, be it from a cardiologist, general practitioner, or nurse. The results are listed (along with $95 \% \mathrm{CI}$ ) in Table 2:

Table 2: Relative risk analysis over 1 year of observations at various time intervals

\begin{tabular}{|c|c|c|c|c|c|}
\hline & 0 to 14 days & 15 to 30 days & $0-30$ days & 31 to 180 days & Overall \\
\hline $\begin{array}{l}\text { Age }(>65 \\
\text { years vs. } \\
\text { under } 65)\end{array}$ & $\begin{array}{l}9.12 \\
(95 \% \text { CI } 1.10, \\
75.0 .)\end{array}$ & $\begin{array}{l}2.02 \\
(0.50,8.21)\end{array}$ & $\begin{array}{l}2.26 \\
(0.96,5.29)\end{array}$ & $\begin{array}{l}0.90 \\
(0.57,1.43)\end{array}$ & $\begin{array}{l}0.89 \\
(0.55,1.45)\end{array}$ \\
\hline $\begin{array}{l}\text { Sex (male vs. } \\
\text { female) }\end{array}$ & $\begin{array}{l}1.13 \\
(0.25 \\
5.0284)\end{array}$ & $\begin{array}{l}0.87 \\
(0.22,3.43)\end{array}$ & $\begin{array}{l}0.998 \\
(0.64,1.54)\end{array}$ & $\begin{array}{l}1.20 \\
(0.76,1.88)\end{array}$ & $\begin{array}{l}0.74 \\
(0.44,1.26)\end{array}$ \\
\hline $\begin{array}{l}\text { Race (white } \\
\text { vs. other) }\end{array}$ & $\begin{array}{l}0.17 \\
(0.04,0.70)\end{array}$ & $\begin{array}{l}0.58 \\
(0.15,2.27)\end{array}$ & $\begin{array}{l}0.44 \\
(0.22,0.83)\end{array}$ & $\begin{array}{l}0.97 \\
(0.57,1.66)\end{array}$ & $\begin{array}{l}0.73 \\
(0.19,2.73)\end{array}$ \\
\hline $\begin{array}{l}\text { NYHA class } \\
\text { ( } 2 \& 3 \text { vs. } 4)\end{array}$ & $\begin{array}{l}0.11 \\
(0.02,0.53)\end{array}$ & $\begin{array}{l}0.60 \\
(0.07,5.11)\end{array}$ & $\begin{array}{l}0.24 \\
(0.09,0.61)\end{array}$ & $\begin{array}{l}1.03 \\
(0.48,2.20)\end{array}$ & $\begin{array}{l}0.78 \\
(0.38,1.57)\end{array}$ \\
\hline $\begin{array}{l}\text { AHA } \\
\text { Compliance } \\
(1 \text { vs. } 0)\end{array}$ & $\begin{array}{l}0.23 \\
(0.86,22.82)\end{array}$ & $\begin{array}{l}1.95 \\
(0.36,11.11)\end{array}$ & $\begin{array}{l}0.79 \\
(0.44,1.44)\end{array}$ & $\begin{array}{l}0.85 \\
(0.53,1.32)\end{array}$ & $\begin{array}{l}0.65 \\
(0.34,1.02)\end{array}$ \\
\hline
\end{tabular}

Relative risk of rehospitalization among elderly individuals (over 65 years) was greatly increased in the first 30 days of enrollment $(\mathrm{RR} 0-14$ days $=9.12, \mathrm{RR} 15-30$ days $=2.02$ ), with later time periods reflecting a lower relative risk than the under 65 years group. This result can commonly be attributed to a survival effect, with worse prognosis among older individuals with multiple hospitalizations. Men, although exhibiting a higher 
relative risk of rehospitalization within the first two weeks of the study period, had an overall lower relative risk of rehospitalization ( $R R$ 0.74) compared to women in the study. Additionally, white individuals had a lower overall relative risk of rehospitalization compared to other ethnicities; in particular, white individuals exhibited an almost $90 \%$ risk reduction relative to other ethnicities in the $0-14$ day rehospitalization period (RR 0.11).

NYHA classes II and III patients relative to class IV patients had a substantially reduced risk of rehospitalization, especially within the first two weeks of enrollment (RR $0.11 ; 95 \% \mathrm{CI} 0.02,0.53)$. The trend can be seen in each time period where risk was calculated, with the an overall risk reduction of $22 \%$ in class II and III patients compared to the class IV patients. The most important factor, however, was the risk among those in which treatment guidelines were not met. Among those individuals in which guideline compliance was met, a $36 \%$ decrease in risk was exhibited over the entire study period compared to those in which treatment guidelines were not met $(\mathrm{RR} 0.63 ; 95 \% \mathrm{CI} 0.34$, $1.02 ; \mathrm{p}=0.06$ ). The first two weeks of enrollment in particular proved to substantially increase the risk of rehospitalization $(\mathrm{RR}=0.23 ; 95 \% \mathrm{CI} 0.04,1.16)$. It should be noted that while not all of the relative risks calculated reached statistical significance at the $\mathrm{p}=0.05$ level, these estimates are of clinical importance due to their representation of a real (randomized) population of individuals with HF.

Because the cohort was monitored from baseline enrollment to 1 year after, the appropriate determination of the importance of treatment guideline compliance was to perform a survival analysis on the subjects, looking at those in which guidelines were met and those in which guidelines were not complied with. To account for interactions among 
multiple covariates, a Cox regression analysis was performed using SPSS (v. 21) to model the relative survival among individuals suffering a CV-related hospitalization or HF-related hospitalization looking at variability among NYHA class and ACC/AHA guideline compliance. Univariate regression models were run for each of the 5 variables of interest: age (classified in 2 groups: over/under 65 years old), race, sex, NYHA class (class IV vs. class II and III), and ACC/AHA compliance. From the univariate analysis, the log-rank test was performed to determine survival differences among the groups in each variable. From these analyses, NYHA and treatment compliance both exhibited a strong effect on failure rates. Age did not reach statistical significance $(\mathrm{p}=0.232)$ in the univariate regression analysis, but was added to the multivariate model due to the older population of individuals used in the study (mean age $=65$ years). Race and sex did not provide a strong indication of any risk differences and were excluded from the final model.

A baseline Cox Proportional Hazards model was created looking at rehospitalization for CV or HF-related causes, and was stratified by those individuals in compliance with ACC/AHA treatment guidelines and those not in compliance; to negate differences which may be created by differences in the quality of treatment received, study arm was used as a control variable. Age and NYHA were both kept dichotomous, with the age cut point at the mean for the study population. The baseline regression equation is listed below:

$$
\log _{e} \hat{\varphi}=\beta_{1} \text { Age }+\beta_{2} \text { NYHAClass }+\beta_{3} \text { AHA Compliance }+\beta_{4} \text { Study Arm }
$$


Likelihood ratio testing was used to evaluate the parameters of the suggested survival model. Interaction terms were tested at various iterations, with no interactions proving a strong effect in which they would be adequately added to the survival model without statistical power. Overall, survival analysis with the baseline model was run looking at the associated hazard rates in the listed variables for different time periods: 0-365 days (overall), $0-14$ days, $15-30$ days, $0-30$ days, $31-180$ days, and $0-180$ days; the plots are listed below, along with their cumulative hazard ratios.

Cumulative Hazard (0-365 days)

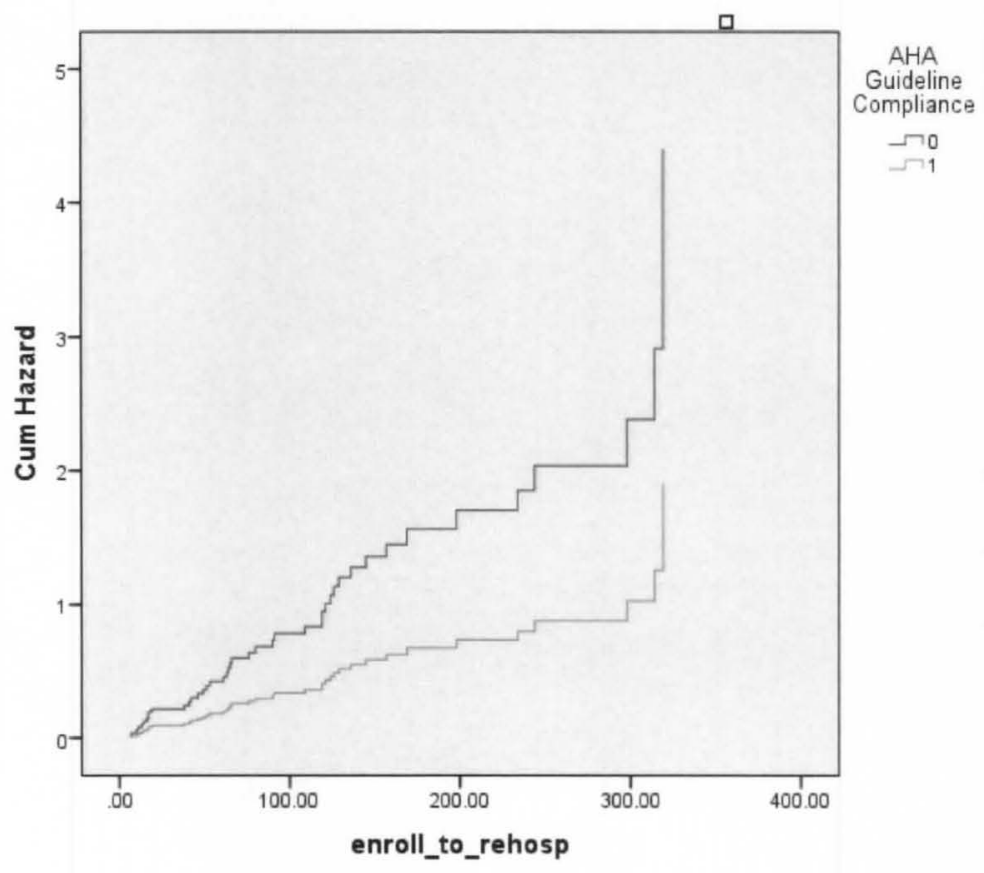

HR: $0.40(95 \% \mathrm{CI}$ :

$0.20,0.81)$

$\mathrm{p}=0.01$ 
Cumulative Hazard 0-14 days:

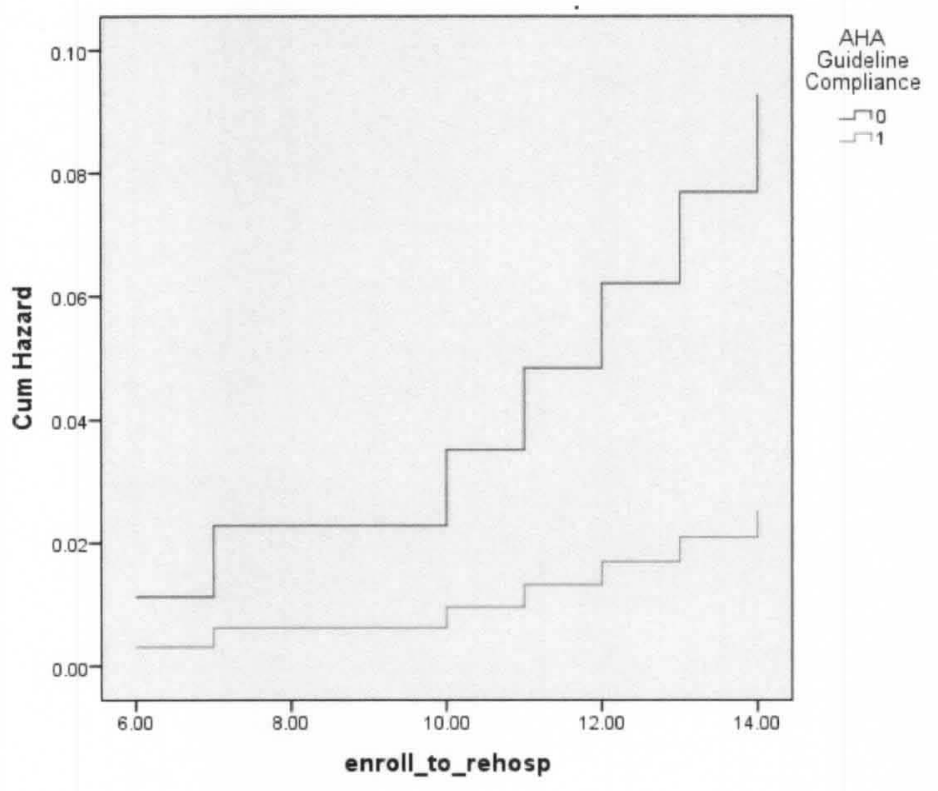

HR: $1.59(95 \% \mathrm{CI}$

$0.33,9.71)$

$\mathrm{p}=0.62$

Cumulative Hazard 15-30 days:

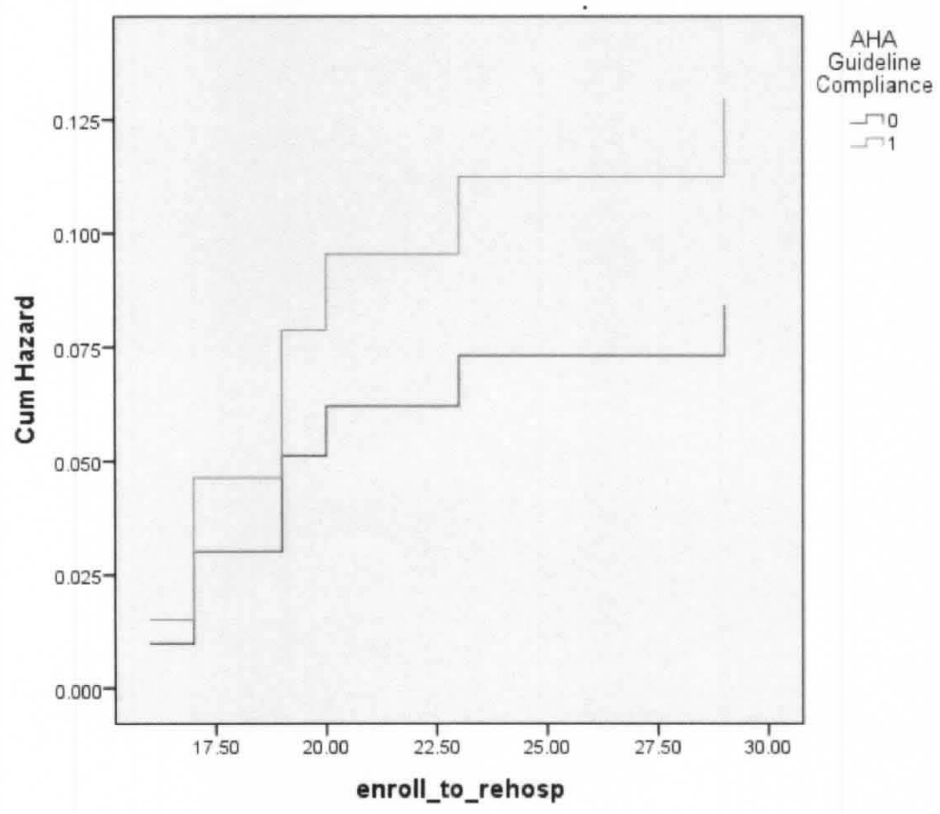

HR: $1.59(95 \% \mathrm{CI}$

$0.33,9.71)$

$\mathrm{p}=0.62$ 
Cumulative Hazard 0-30 days

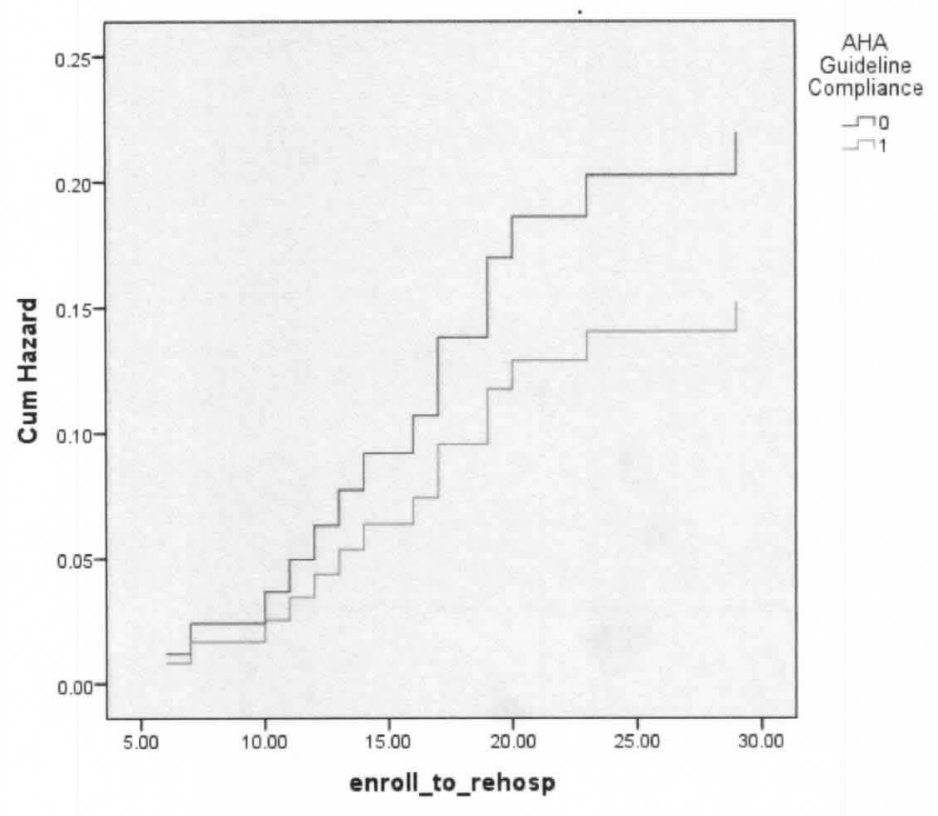

HR: 0.68 (95\%CI 0.21, 2.33)

$\mathrm{p}=0.54$

Cumulative Hazard 31-180 days:

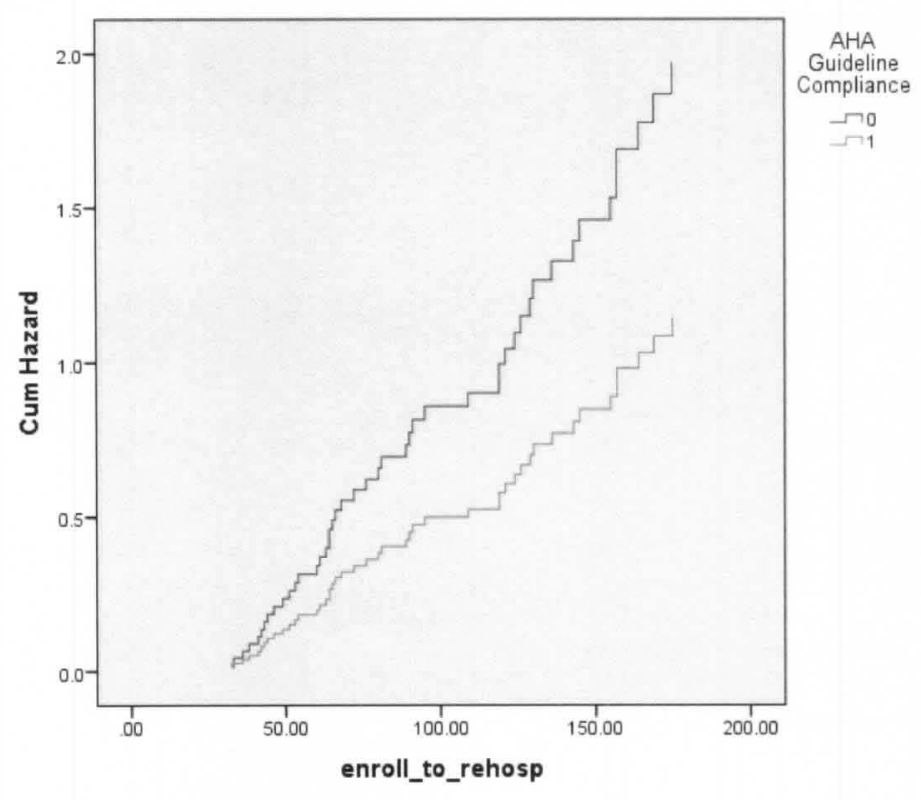

HR: 0.57 (95\%CI 0.29, 1.12)

$\mathrm{p}=0.11$ 
Cumulative Hazard 0-180 days:

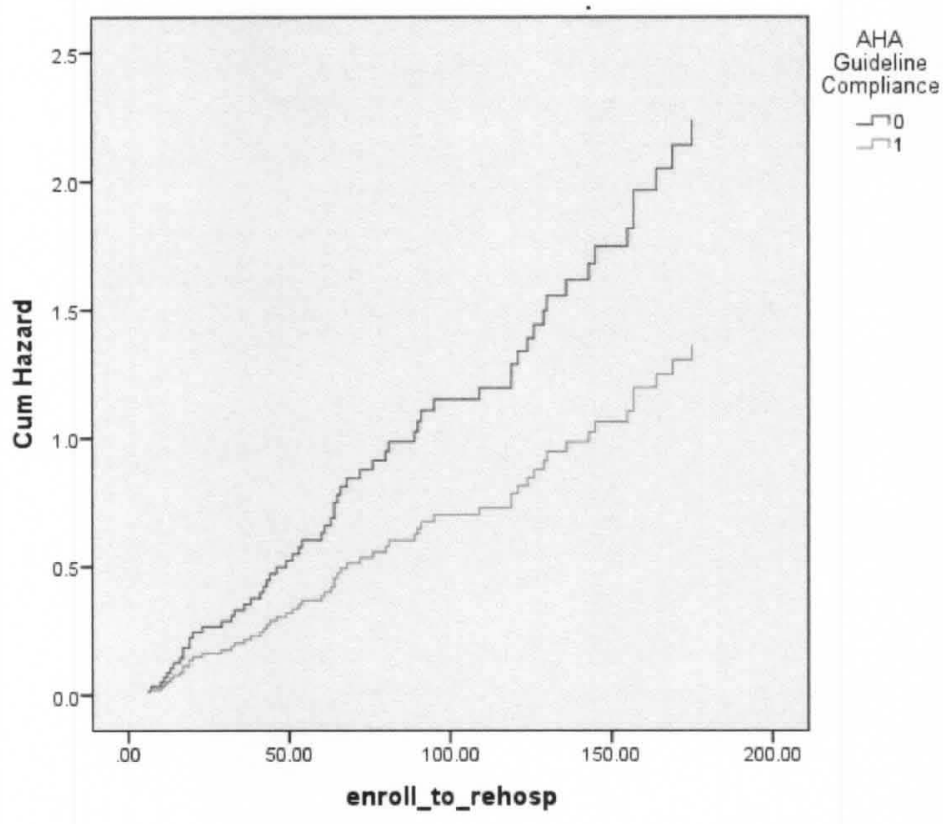

HR: 0.61 (95\%CI 0.34,

1.09)

$\mathrm{p}=0.09$ 


\section{CONCLUSIONS:}

Over the course of the year in which the study was conducted, a decreased hazard rate (HR: $0.40 ; 95 \% \mathrm{CI}: 0.20,0.81 ; \mathrm{p}=0.01$ ) was observed in patients in which ACC/AHA treatment guidelines were met. The largest hazard rate decrease was experienced within the first 2 weeks of patient enrollment, with relative hazard increasing over time; this suggests that guideline compliance is particularly important to the immediate health of a patient with heart failure, no matter the severity of heart failure (as designated by NYHA class). With the exception of the $15-30(\mathrm{HR}-15$ to 30 day $=1.59, \mathrm{p}=0.62)$ day period, a decreased hazard rate was observed for each of the specified time periods. Observed risk variances exhibited may be attributed to different phases of HF over the study period, with individuals failing early at more severe stages of $\mathrm{HF}$ than those at the later stages of the study. In addition, multiple failures over the study period without having a HF-related rehospitalization may contribute to the observed variances in risk.

Individuals in NYHA class IV were at an increased risk of rehospitalization relative to those in class II and III, which is expected based on the criteria for classification, but especially within the first 30 days of enrollment. Cumulative hazard decreases ranged between $20-40 \%$ among those receiving appropriate treatment therapies, suggest that appropriate treatment from the outset of hospitalization conferring even a small increase in health outcomes would provide a dramatic benefit, given the relative expense associated with a single heart failure hospitalization. The results of the 
analysis argue the importance of treatment adherence among heart failure patients, no matter what stage of heart failure (but especially in the most severe stage and within the first 30 days.

The original study, enrolling patients from medical institutions in multiple regions of the United States, collected a random sample of individuals representative of the US population. The main limitation of the analysis of this data is the small sample size $(\mathrm{n}=128)$, resulting in lack of power to detect statistical significance. The limited sample size reduced the ability to make a fully inclusive model integrating several demographic characteristics; race and sex, which were tested in univariate analysis, were dropped from later analyses due to a lack of effect. Comorbid conditions (i.e., hypertension, diabetes, hypercholesterolemia, hyperlipidemia, obesity, and smoking status) which were recorded at enrollment for each patient were excluded from regression models to limit the number of variables used in the analyses. To account for the health implications of these comorbidities and their effects on an individual's overall survival probability, regression analysis proceeded under the assumption that patients with moderate to severe heart failure were receiving appropriate interventions and counseling for lifestyle modification for their accompanying health risks (such as diabetes, hypertension, obesity and smoking)- which typically interact negatively with the heart.

In addition, the argument for appropriate treatment was simplified for the purposes of analysis, using an all-or-none approach. In future studies, a stratification of partial adherence to guidelines could be used to indicate any weaknesses in a specific area of heart failure treatment, which extends beyond the scope of this research (ex. Presence of hypokalemia and adverse interactions with spiranolactone). Of greater 
importance is the use of an intention-to-treat model in the original study, which assumes a patient complies with the recommended treatments; a study assessing medication intake at each rehospitalization event may provide a more accurate depiction of the hazard rates in these heart failure patients, setting apart those that follow prescribed treatment with those that do not.

Given the overall mortality rate among heart failure patients, the results from the regression analysis should be assessed on the basis of their clinical implications, considering the relative risks and survival effects among the patients enrolled. The small sample size $(\mathrm{n}=128)$ did not achieve statistical significance for more complex models including variables such as comorbid conditions, as stratification often lead to small subcategories. Because of this, a simplified model was created to account for the lack of power. The effect size (decreased hazard among those in which treatment guidelines were adhered) solely due to treatment is a large estimate which should be considered of clinically meaningful importance, due to an already increased risk of failure in the target population. The confidence interval in the overall model, suggest a positive effect in this patient population. Given that the study population was a representation of a diverse group of heart failure patients, the effect size of treatment can be extrapolated to the larger population of heart failure patients. Effect size within the immediate time after enrollment into the study suggests that compliance with the set $\mathrm{ACC} / \mathrm{AHA}$ guidelines from the outset of heart failure would improve immediate rehospitalization rates, which has both a workload benefit as well as a monetary benefit to the medical institutions receiving these patients. Additionally, since heart failure treatment is one of the largest 
expenditures in healthcare, even a small reduction in overall mortality would benefit our healthcare system on a large scale.

\section{DISCUSSION:}

Previous studies looking at the relationship between treatment and rehospitalization rates among heart failure patients have concluded similar results, arguing that appropriate treatment reduces the rates in which a patient is readmitted; the implication, although intuitive, is often understated based on the cumulative risk among these patients. What makes this analysis unique is that importance of appropriate treatment has been assessed not only on a larger time scale (1 year), but it has also analyzed the effect at various smaller time frames: 0-14 days, 15-30 days, and 31-180 days. These time frames not only have a clinical importance, but an economic importance. As stated previously, heart failure is one of the most expensive conditions in terms of overall healthcare spending, accounting for approximately $2 \%$ of total costs (estimated at 37 billion dollars). With the initiation of the Affordable Care Act (ACA), hospitals and medical institutions will now incur penalties based on the percentage of rehospitalizations assessed over these shorter time periods.

The Centers for Medicare and Medicaid Services (CMS's), in response to limited improvement of 30 day rehospitalization rates between 2007 and 2010, implemented a National Strategy for Quality Improvement in Health Care, the goal of this program aiming to reduce hospitalizations by $20 \%$ at the end of this year. CMS's estimated that such a reduction would prevent 1.6 million hospitalizations and save an estimated $\$ 15$ billion. The most important program in the effort to reduce rehospitalization is the 
Hospital Readmission Reduction Program (HHRP), specified in the bylaws of the ACA. This program, which is expected to generate significant savings in the Congressional Budget, is designed to align payment with outcomes, driving meaningful reductions in all-cause readmissions. The measure proposed is a hospital-specific, risk-standardized 30 day readmission ratio which follows index admissions for acute myocardial infarction (AMI) and HF. As a result, underperforming hospitals will incur a reduction of $1 \%$ or less in Medicare reimbursements for inpatient services; payment penalties can increase to $2-3 \%$, based on the higher readmission rates. This would translate into an average cost per hospital of $\$ 125,000 .^{4}$ Hospital readmission rates, based on 2010 data would have placed half of all eligible hospitals at risk of penalties regardless of geography, bed size, or teaching status.

The issue of proper treatment and penalty for readmission is one that has to be weighed from the sides of the physicians and clinicians, and not that of the agencies working to reduce rehospitalization rates. A couple of issues to contemplate with the proposed hospital readmissions reduction program include higher readmissions rates among hospitals serving low-income individuals, and hospitals keeping very ill patients having higher readmissions/lower mortality. In addition, heart failure mortality may not be the direct cause of readmission; any number of maladies or physical problems can arise sending someone back to the hospital. The $20 \%$ reduction goal placed by CMS is based on the average rate of rehospitalization among Medicare recipients within the first month after hospital discharge for any purpose; however, readmission for only a limited number of conditions (HF included) would be recorded when assessing penalties accrued. From this study, it is clear that age, HF class, and especially treatment guideline 
adherence is an important determinant in the first 30 days; this is exhibited by an increase in relative risk among age and NYHA class, and a decrease with treatment compliance (listed previously in Table 2) as well as by the cumulative 30 day hazard reduction of almost $35 \%$. By the measures proposed, hospitals would benefit from focusing better treatment on patients with heart conditions relative to all other conditions.

The guidelines for clinical practice set forth by the ACC/AHA are recommendations based on the amount of clinical evidence backing certain therapies. They are categorized by the amount of data on (level A-C) and effect size (class) of treatment. While these guidelines are widely used and accepted by clinicians and physicians, they are not all encompassing, as treatment for heart failure is complex. The guidelines are set to be a baseline for treatment, however several clinical and biological measures are often assessed upon patient examination which may modify the course of medical action. From an epidemiological perspective, however, these levels of evidence are something that could be translated into the evaluation of research outside of the clinical realm. The possibility of having a standardized approach for research evaluation in different areas of epidemiology, using reinforcement from multiple studies create an "evidence-based" methodology could be beneficial in making recommendations in the general public.

In conclusion, from this analysis we have assessed the economic and clinical benefits of appropriate treatment for heart failure patients. Looking at various factors that influence the overall rehospitalization rates including the severity of heart failure, treatment compliance is arguably the most important variable within the results generated. While the sample of patients was small in this study, the large effect - 
exemplified by hazard rates among those receiving appropriate treatment - argues the clinical importance of adhering to the ACC/AHA guidelines, while controlling for several other factors that contribute to increased risk. Further studies with a larger sample size would be needed to reaffirm the finding from this analysis with a strong statistical significance. With the initiation of the Affordable Care Act, however, there is even more of an incentive for hospitals to become thorough in their efforts to properly treat these high risk patients, and the results from our study reaffirm this point. 


\section{REFERENCES:}

1. American Heart Association "About Heart Failure", August 20, 2013. $<$ http:/www.heart.org/HEARTORG/Conditions/HeartFailure/AboutHeartFailure/ $. j \mathrm{sp}>$

2. Barker WH, Mullolly JP, Getchell W. Changing incidence and survival for heart failure in a well-defined older population, 1970-1974 and 1990-1994. Circulation, 2006; 113:799-805

3. Bauersachs J, Heck M, Fraccarollo D, et al. Addition of spironolactone to angiotensin-converting enzyme inhibition in heart failure improves endothelial vasomotor dysfunction. Journal of the American College of Cardiology, 2002; 39(2):351-358.

4. Bogaev, RC. Cost considerations in the treatment of heart failure. Texas Heart Institute Journal, 2010; 37(5):557-558.

5. Bozkurt B, Agoston I, Knowlton AA. Complications of inappropriate use of spironolactone in heart failure: When an old medicine spirals out of new guidelines. Journal of the American College of Cardiology, 2003; 41(2):211-214.

6. Centers for Disease Control and Prevention "National Health and Nutrition Examination Survey" <http://www.cdc.gov/nchs/nhanes.htm>

7. Cohn $\mathrm{N}$ and Tognoni G. A randomized trial of the angiotensin-receptor blocker valsartan in chronic heart failure. NEJM, 2001; 345(23):1667-1675.

8. Doughty RN, Rodgers A, Sharpe N, et al. Effects of beta-blocker therapy on mortality in patients with heart failure. European Heart Failure, 1997; 18:560565.

9. Dunlay SM, Shah ND, Shi Q, et al. Lifetime costs of medical care after heart failure diagnosis. Circ Cardiovascular Qual Outcomes, 2011; 4(1):68-75.

10. Eriksson H. Heart failure: A growing public health problem. Journal of Internal Medicine, 1995; 237:135-141.

11. Ezekowitz J, McAlister FA, Humphries $\mathrm{KH}$, et al. The association among renal insufficiency, pharmacotherapy, and outcomes in 6,427 patients with heart failure and coronary artery disease. Journal of the American College of Cardiology, 2004; 44(8):1587-92.

12. Flather MD, Yusuf S, Kober L, et al. Long-term ACE-inhibitor therapy in patients with heart failure of left-ventricular dysfunction: a systematic overview of data from individual patients. Lancet, 2000; 355:1575-1581. 
13. Gambassi G, Lapane KL, Sgadart A, et al. Effects of angiotensin-converting enzyme inhibitors and digoxin on health outcomes of very old patients with heart failure. Archives of Internal Medicine, 2000; 160:53-60.

14. Hunt SA, Baker DW, Chin MH et al. ACC/AHA guidelines for the evaluation and management of chronic heart failure in the adult: Executive summary a report of the American College of Cardiology/American Heart Association task force on the practice guidelines (committee to revise the 1995 guidelines for the evaluation and management of heart failure). Circulation, 2001; 104:2996-3007.

15. Kalon KL, Pinsky JL, Kannel WB, et al. The epidemiology of heart failure: The Framingham study. JACC, 1993; 22(4):6A-13A.

16. Kochanek KD, Xu J, Murphy SL, et al. "National Vital Statistics Report, Vol 20, No. 3." 29 December 2009. Centers for Disease Control and Prevention. <www.cdc.gov/nchs/deaths.htm>

17. Labarthe, Darwin. Epidemiology and prevention of cardiovascular disease: $a$ global challenge $2^{\text {nd }} E d$. 2010. Sudbury, MA; Jones and Bartlett Publishers.

18. Lee DS, Tu JV, Juurlink DN, et al. Risk-treatment mismatch in the pharmacotherapy of heart failure. JAMA, 2005; 294:1240-1247.

19. Loehr LR, Rosamond WD, Chang PP, et al. Heart failure incidence and survival (from the Atherosclerosis Risk in Communities study). The American Journal of Cardiology, 2008; 101(7):1016-1022.

20. Mayo Clinic "Heart Failure", December 23,2011 $<$ http://www.mayoclinic.com/health/heart-failure/DS00061>

21. McMurray J, Cohen-Solal A, Dietz R, et al. Practical recommendations for the use of ACE inhibitors, beta-blockers, aldosterone antagonists and angiotensin receptor blockers in heart failure: Putting guidelines into practice. The European Journal of Heart Failure, 2005; 7:710-721.

22. Packer M, Coats AJS, Fowler MB, et al. Effect of carvedilol on survival in severe chronic heart failure. The New England Journal of Medicine, 2001; 344(22):1651-1658.

23. Pitt B, Poole-Wilson PA, Segal R, et al. Effect of losartan compared with captopril on mortality in patients with symptomatic heart failure: randomized trial - the Losartan Heart Failure Survival Study ELITE II. The Lancet, 2000; 355:1582-1588.

24. Pitt B, Segal R, Martinez FA, et al. Randomised trial of losartan versus captopril over 65 with heart failure (Evaluation of Losartan in the Elderly Study, ELITE). Lancet, 1997; 349:747-752.

25. Pitt B, Zanniad F, Remme WJ, et al. The effect of spironolactone on morbidity and mortality in patients with severe heart failure. NEJM, 1999; 341(10):709717. 
26. Raphael C, Briscoe C, Davies J, et al. Limitations of the New York Heart Association functional classification system and self-reported walking distances in chronic heart failure. Heart, 2007; 93:476-482.

27. Roger V, Go AS, Lloyd-Jones DM, et al. Heart disease and stroke statistics-2012 update. Circulation, 2012; 125:2-220.

28. Shibata MC, Flather MD, Wang D. Systematic review of the impact of beta blockers on mortality and hospital admissions in heart failure. The European Journal of Heart Failure, 2001; 3:351-357. 


\section{CURRICULUM VITAE:}

Matthew Thomas Mefford

1726 S. Second St., Apt 12

(502) 744-6894

Louisville KY, 40208

mtmeff01@cardmail.louisville.edu

Profile:

- Highly motivated student seeking professional experience in the area of epidemiology, public health, and data analysis with the intent of pursuing a doctoral degree

Research Interests:

- Cardiovascular disease epidemiology; evidence-based medicine; critical evaluation of emergency care; chronic disease epidemiology

Skills:

- Collect and analyze data using SAS, R, and Excel software.

- Research and solve problems

- Write reports and present findings

- Explain complex information to a wide range of audiences

- Work well in team environments to accomplish tasks efficiently

Relevant Academic Work:

- Thesis:

- "Adherence to ACC/AHA guidelines for pharmacotherapy treatment in heart failure patients and associated disparities in rehospitalization rates." Anticipated April 2013

- Independent Study

o "Impact of treatment on perceived pain in breast cancer survivors" - Fall 2012

- "Locating and inspecting automated external defibrillators in shopping malls in the city of Louisville" - Fall 2012

Education:

- University of Louisville School of Public Health and Information Sciences Louisville, KY 40202

Epidemiology

August 2011 -PresentM.S.

Cumulative GPA: 3.9 Anticipated graduation May 2013 
- University of Louisville

Louisville, KY 40202

B.S. Biology

August 2007-May 2011

Cumulative GPA: 3.1

Graduated May 2011

Work Experience:

- The Brown Hotel (May 2010 - Present)

- Front Desk Associate

- Employee of the quarter: Fall 2011

- Providing exceptional guest services in an upscale, luxury hotel

- Checking availability and creating hotel reservations

- Assisting with a wide range of concierge tasks

- Coordinating requests between guests and other hotel departments as the operator

- United Parcel Service (July 2007-June 2010)

- Package Handler

- Work with others in a fast-paced, busy environment, under physically demanding conditions

- Load packages into air containers, keeping delivery flights on schedule

- Hazardous Materials training/certification

Awards

- Graduate Dean's Citation - April 2013

Organization/Group Involvement:

- Kentucky Academy of Science - member

- GRASS - Student Sustainability Organization, UofL - member

- commonGround - LGBT /straight alliance - member 